\title{
Magnetic monopole search with the MoEDAL test trapping de- tector
}

\author{
Akshay Katre (On behalf of the MoEDAL Collaboration) $)^{1, a}$ \\ ${ }^{1}$ Particle physics department, University of Geneva, 1211 Geneva 4, Switzerland
}

\begin{abstract}
MoEDAL is designed to search for monopoles produced in high-energy Large Hadron Collider (LHC) collisions, based on two complementary techniques: nucleartrack detectors for high-ionisation signatures and other highly ionising avatars of new physics, and trapping volumes for direct magnetic charge measurements with a superconducting magnetometer. The MoEDAL test trapping detector array deployed in 2012, consisting of over 600 aluminium samples, was analysed and found to be consistent with zero trapped magnetic charge. Stopping acceptances are obtained from a simulation of monopole propagation in matter for a range of charges and masses, allowing to set modelindependent and model-dependent limits on monopole production cross sections. Multiples of the fundamental Dirac magnetic charge are probed for the first time at the LHC.
\end{abstract}

\section{Introduction}

Magnetic monopoles were first postulated by Dirac in 1931, who showed that with their existence, electric charge quantisation could be explained as a natural consequence of angular momentum quantisation [1]. Their introduction would also add symmetry to Maxwell's equations of electromagnetism. 't Hooft and Polyakov, in 1974, independently demonstrated that a Grand Unified Theory (GUT) scheme possesses a monopole solution when a U(1) subgroup of electromagnetism that is embedded into a larger gauge group is spontaneously broken by the Higgs mechanism [2, 3]. Monopole solutions have been proposed to arise within the electroweak theory itself [4], which relies on spontaneous gauge symmetry breaking. The Cho-Maison electroweak monopole would have a mass of the order of several $\mathrm{TeV}[5,6]$ and is possibly within the range of the Large Hadron Collider (LHC). With the abundance of monopole theories and no definite estimate on its mass, the search for free magnetic charges in nature is compelling.

Dirac proposed that the monopoles should carry a magnetic charge $g$ equal to the multiple of a fundamental unit of magnetic charge referred to as the Dirac charge $g_{D}: g=n \cdot g_{D}$, where $g_{D}$ is equivalent to 68.5 times the charge of an electron. The minimum value of the quantisation number $n$ varies between different theories. According to Dirac, $n=1,2,3$.., while according to Schwinger, as well as, Cho and Maison, $n=2,4,6$. [ [4, 7], and $n=3,6,9$.. or $n=6,12,18$.. if one considers the elementary charge to be carried by the down quark. As a result of the high value of the Dirac magnetic charge, a high velocity monopole is expected to suffer energy losses in matter over $\approx 4700$ times higher than a muon [8-10]. Thus, in general monopoles in nature will manifest themselves as

\footnotetext{
a e-mail: akshay.k@cern.ch
} 
highly ionising particles. A free magnetic charge will also induce a persistent current when passed through a superconducting loop. Searches for monopoles using these two signatures (high ionisation and magnetic induction) were performed in cosmic rays and in matter, and at accelerators each time a new energy regime was reached [11].

At colliders, three kinds of techniques are commonly used to search for magnetic monopoles: (1) General-purpose detectors with high ionisation energy loss detection capabilities (e.g. OPAL at LEP [12] and CDF at the Tevatron [13]); (2) dedicated deployment of nuclear-track detectors [14] around the interaction points (e.g. at LEP [15, 16] and at the Tevatron [17]); and (3) the induction technique applied to accelerator and detector material in which monopoles have stopped and remained trapped (e.g. at HERA [18] and at the Tevatron [19, 20]). These searches have excluded the presence of monopoles with charges equal to or above the Dirac charge and masses up to $400 \mathrm{GeV}$. Masses higher by one order of magnitude can be probed at the LHC. For complementary searches, the LHC programme should include all three of these techniques [21]. The general-purpose ATLAS detector has performed searches for monopoles at 7 and $8 \mathrm{TeV}$ centre of mass energies [22, 23]. The MoEDAL experiment near the LHCb interaction point uses a combination of in-flight detection with nucleartrack detectors and re-usable aluminium trapping detectors [24].

\section{Experimental setup}

The Monopole \& Exotics Detector at the LHC (MoEDAL) experiment is a dedicated experiment designed to enhance, in a complementary way, the physics reach of the LHC. The primary objectives of MoEDAL is to directly search for the Magnetic Monopole or Dyon and other highly ionising Stable (or meta- stable) Massive Particles (SMPs) at the LHC [25].
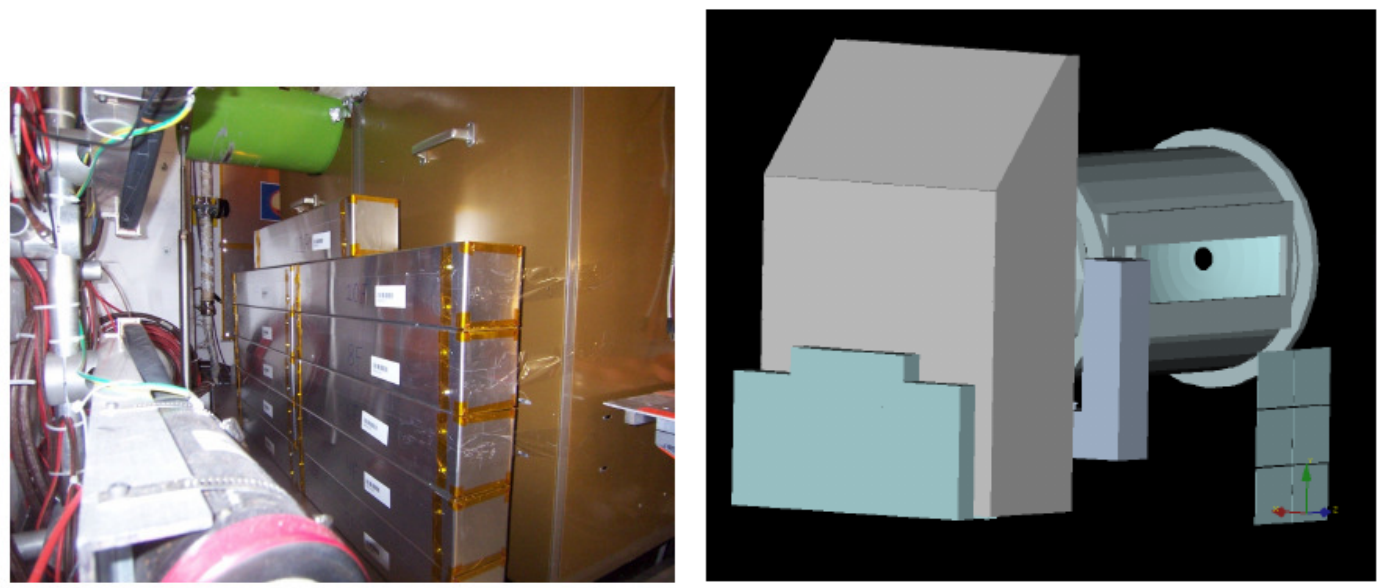

Figure 1. Left: Photograph of the 2012 MMT test array placed downstream of the LHCb stacked under the beam pipe $1.8 \mathrm{~m}$ away from the interaction point along the back of the VELO vacuum vessel. Right: Simulation of the LHCb cavern with the MMT array [26, 27].

Deployed around the LHCb experiment's Vertex Locator (VELO) cavern, it is a unique and largely passive LHC detector that comprises four sub-detector systems. The largest sub-detector systems are arrays of two nuclear-track detectors which are optimised to probe a range of particle charges. The 
TimePix pixel devices form a real-time radiation monitoring system devoted to monitoring of highlyionising backgrounds in the MoEDAL cavern. The Magnetic Monopole Trapper (MMT) is the other passive sub-detector system that is capable of capturing long-lived charged particles and measure directly magnetic charge properties of particles.

\subsection{MMT sub-detector system}

The MoEDAL MMT test detector system is an aluminium volume placed in the vicinity of the LHCb interaction point. Aluminium is a good choice for the trapping volume material for three important reasons. First, the anomalously large magnetic moment of aluminium nucleus means that it will strongly bind a trapped monopole. Second, aluminium does not present a problem with respect to activation. Lastly, aluminium allows a cost effective approach to the construction of the MMT detector. In September 2012, an initial MMT test array comprising 11 boxes each containing 18 rods of $60 \mathrm{~cm}$ length and $2.5 \mathrm{~cm}$ diameter was installed and exposed to $0.75 \mathrm{fb}^{-1}$ of $8 \mathrm{TeV}$ proton-proton collisions. The boxes were placed downstream of the $\mathrm{LHCb}$ detector, $1.5 \mathrm{~m}$ away from the interaction point, immediately in front of the LHCb VELO vacuum vessel, covering 1.3\% of the total solid angle (Fig. 1). After the completion of the run, the rods are cut into samples of $20 \mathrm{~cm}$ length (except for samples

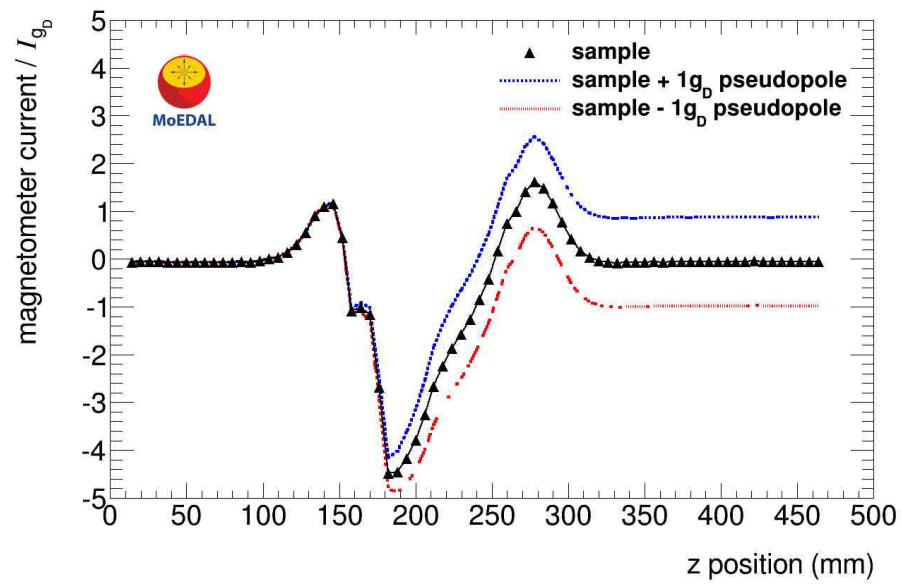

Figure 2. The plot shows the response of the magnetometer current at 76 different positions for a single sample as it traverses through the magnetometer. The dashed blue and red lines indicate the expected response from the magnetometer if a magnetic monopole of charge +1 and -1 $g_{D}$ is trapped in the sample.

from box 11, which were cut into a mix of 10, 15, 20 and $30 \mathrm{~cm}$ samples), which total to 606 samples that are later passed through the magnetometer for measurements.

A DC-SQUID rock magnetometer based at the Laboratory of Natural Magnetism (ETH-Zurich) was used. The magnetometer is capable of detecting monopole charges much less and much greater than the Dirac charge as was demonstrated in previous studies [28]. The calibration was performed with a convolution method applied to a dipole sample, and cross-checked using thin solenoids which mimic a monopole of well known magnetic charge [28]. The response of the magnetometer was found to be linear and charge symmetric which allowed to express the measured currents in units of Dirac magnetic charge. Fig. 2 shows the magnetometer current response as one sample traverses through the magnetometer. The dashed lines show the expected response from the sample if a magnetic monopole of charge $\pm g_{D}$.

Each of the samples was passed through the magnetometer at least once over a 7-day measurement campaign in September $2013^{1}$. On average, every tenth measurement was performed with an empty

\footnotetext{
${ }^{1}$ The samples from Box 11 were measured earlier in May 2013
} 


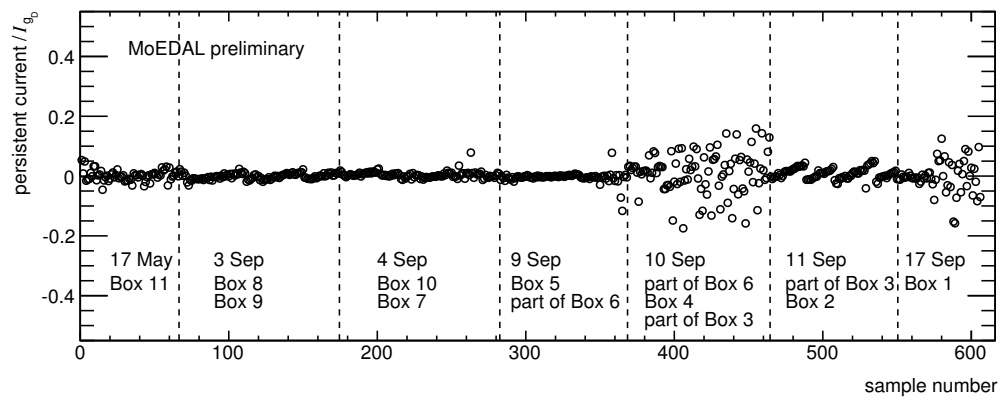

Figure 3. The persistent current (in terms of Dirac charge) measured for 606 samples from the MMT boxes during the September 2013 measurement campaign [29].

sample holder for an offset subtraction. The relevant value is the persistent current, defined as the difference between the currents measured after and before passage of the sample through the sensing coil, and then subtracting the difference obtained with a nearby empty holder measurement (Fig. 3).

Whenever the persistent current differs from zero by more than one fourth of the Dirac charge, the sample is considered as a candidate and measured again several times. A sample that contained a trapped monopole would consistently yield the same value for repeated measurements, while deviations in the first measurement correspond to instrumental effects (Fig. 4). The factors that affect the magnetometer response could be due to flux jumps occurring when the slew rate is large, noise currents in the SQUID feedback loop, small variations in the length of the sample holder from one run to the next and accumulation of condensed water and ice in the magnetometer tube near the cold sensing region.

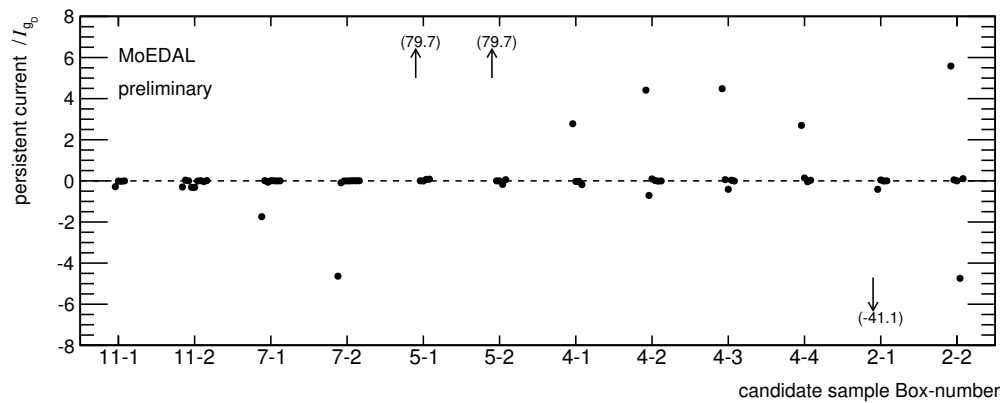

Figure 4. Results of multiple persistent current measurements (in units of Dirac charge) for the samples that yielded large $\left(|g|>0.25 g_{D}\right)$ values for the first measurement [29].

\section{Monopole simulation}

For model-dependent and model-independent interpretations of the results, magnetic monopoles were simulated using Drell-Yan (DY) and Single-monopole production [29]. 
The leading-order DY process produces a pair of heavy monopoles from initial $p p$ state by quarkantiquark annihilation into a virtual photon using MADGRAPH5 Monte-Carlo event generator. The monopole coupling to the $\mathrm{Z}$ boson is set to zero. PYTHIA is used for the initial-state radiation and the hadronisation and the underlying event. The kinematic distributions for the spin- $1 / 2$ monopoles are shown in Fig. 5.
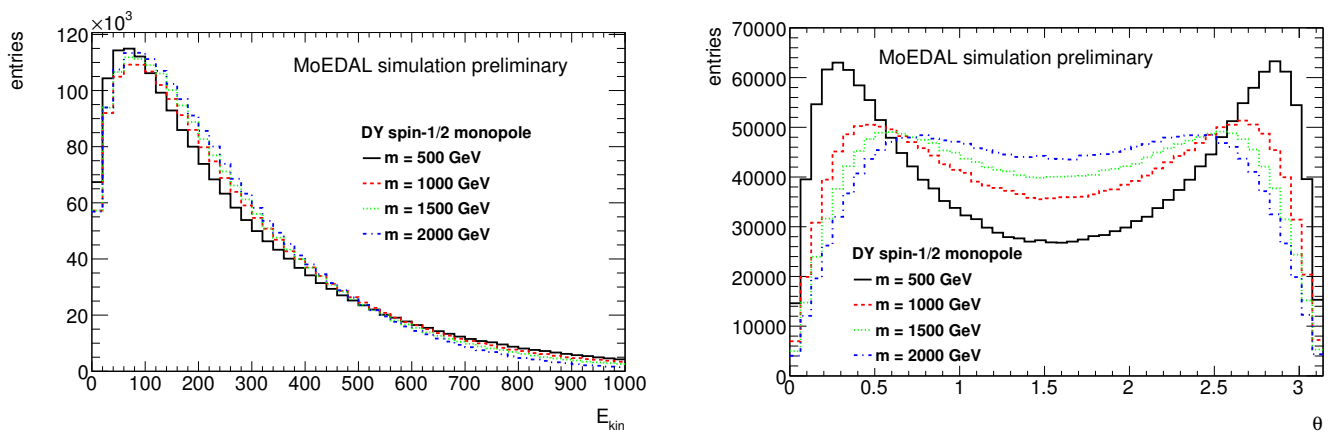

Figure 5. The kinematic distributions of spin-1/2 pair-produced monopoles considered for model-dependent results [29].

The Single-monopole samples are generated with a flat kinetic energy distribution ranging from 0 to $10000 \mathrm{GeV}$ and a flat $\theta$ and $\phi$ distribution which encompasses the angular distribution of the trapping detector, i.e., $2.4 \mathrm{rad}<\theta<3.0 \mathrm{rad}$ and $-2.7 \mathrm{rad}<\phi<-0.5 \mathrm{rad}$.

The acceptance of the trapping detector is defined on an event-by-event basis as the probability that the monopole stops inside one of the aluminium rods ${ }^{2}$. It is determined by propagating monopoles into the geometry model. The acceptance is defined in a unique way such that it only depends on the geometry of the detector and not on the production model. Since the collisions are symmetric with respect to the azimuthal angle $\phi$, only two kinematic variables are needed to define the acceptance in a model-independent manner. The two variables chosen here are the longitudinal kinetic energy $E_{z}^{k i n}$ and the polar angle $\theta$, after restricting the denominator of the acceptance definition to the range -2.7 $\operatorname{rad}<\phi<-0.5 \mathrm{rad}$ (encompassing the extent of the trapping detector). Thus for Single-monopole Monte-Carlo samples, the acceptance is mapped for all mass and charge combinations as a function of $E_{z}^{k i n}$ and $\theta$ (with $-2.7<\theta<-0.5 \mathrm{rad}$ ), as show in Fig. 6 for monopoles with $m=1000 \mathrm{GeV}$. These two-dimensional histograms contain all the information needed to obtain the acceptance in any given pair-production model to a good approximation.

The DY acceptances can be computed in two ways. The first method requires full simulated pair-produced monopoles to be tracked through the geometry of the MoEDAL detector in order to compute the acceptances. The more computationally efficient way is to use the Single-monopole efficiency maps (described above) folded with the DY pair-production kinematics for both spin-1/2 and spin-0 monopoles distributions.

\section{Systematics}

The uncertainty in the amount of material in the geometry description of MoEDAL is the dominant source of systematic error. The VELO region within the acceptance of the LHCb detector is designed

\footnotetext{
${ }^{2}$ In the case of pair-produced DY monopoles, at least one of the two monopoles is required to stop inside the aluminium rods.
} 

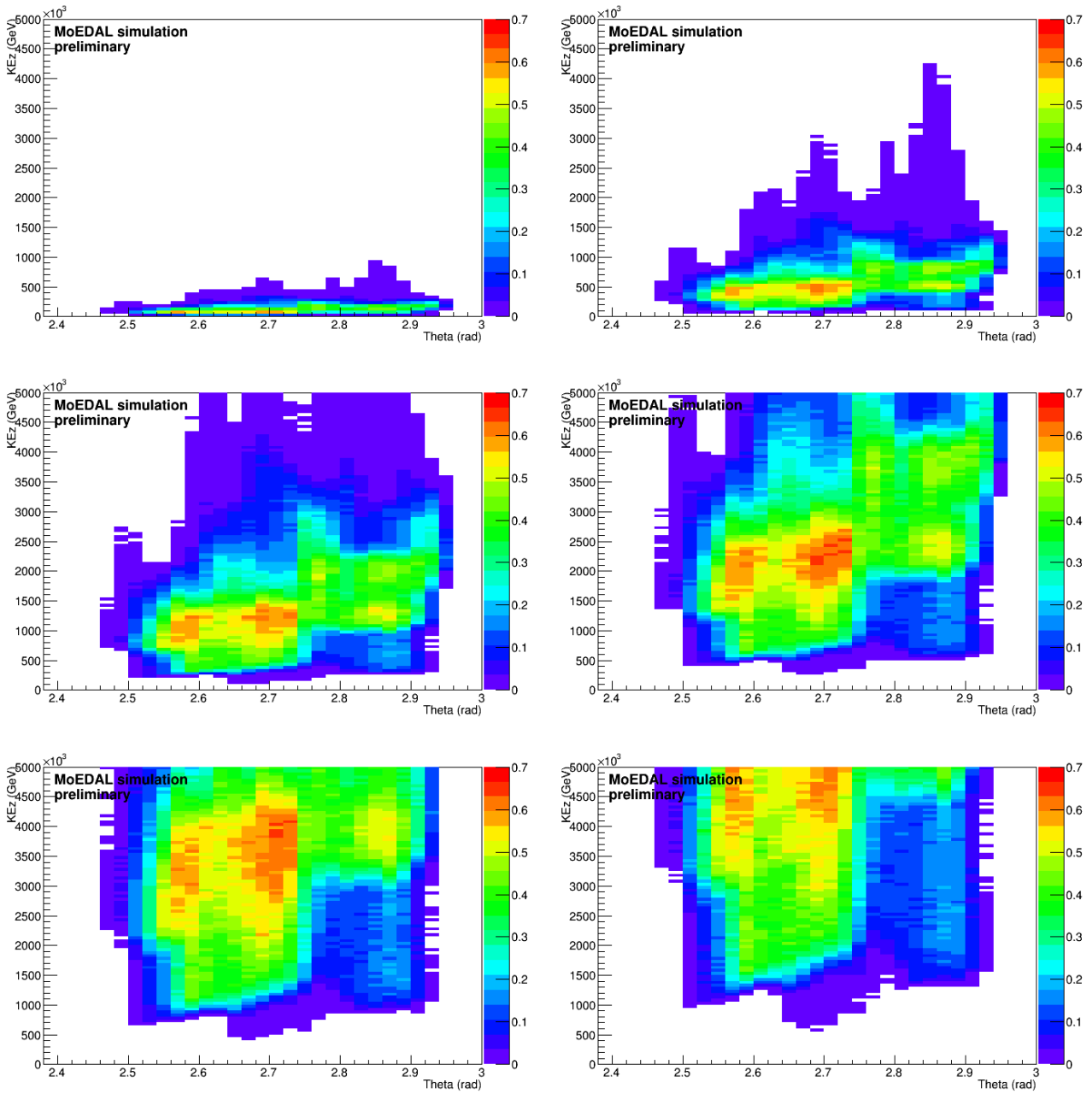

Figure 6. The acceptance of the MoEDAL test array in the kinetic energy and $\theta$ parameter space for monopole charges from $1.0-6.0 \mathrm{gD}[29]$.

to minimise the amount of material obstructing the passage of measured particles. This region has been well modelled by GEANT4. However, the MoEDAL test trapping detector considered here lies just upstream of the VELO vacuum vessel in a more complex region containing: cables, manifolds, and piping, where detailed drawings are not always available. In order to account for possible imperfect understanding of the material in this arena two geometry models were constructed to conservatively describe the minimum and maximum amount of material. These models are based on a detailed physical examination of the material in this region. The uncertainty on the material description has a direct consequence on the acceptance maps, that affects both model-independent and model-dependent results. At the time of writing these proceedings, the determination of these systematic uncertainties is ongoing and will be documented in future publications [29]. 


\section{Results and conclusions}

MoEDAL is designed for passive detection of magnetic monopoles, both in-flight (with the track-etch technique) and trapped (with the induction technique). This is the first time at a collider experiment with a dedicated scalable and recyclable monopole trapping array has been deployed. Under the assumption of monopole capture by aluminium nuclei, there were no magnetic monopoles candidates found in 606 samples of the MMT test array exposed to $0.75 \mathrm{fb}^{-1}$ of $8 \mathrm{TeV}$ proton-proton collisions.

Despite its small acceptance and modest received luminosity MoEDAL's test MMT detector is capable of probing ranges of charge, mass and energy that were not accessed by other LHC experiments. Additionally, this technique can yield results very quickly and would allow for and unambiguous background-free assessment of a signal. A new, larger trapping detector array with a sensitive mass of around 1 tonne was deployed in 2014 in the LHCb cavern, allowing to perform a search in 13 $\mathrm{TeV}$ collisions in the near future.

\section{References}

[1] P.Dirac, Quantised Singularities in the Electromagnetic Field, Proc. Roy. Soc. A 133 (1931) 60.

[2] G. 't Hooft, Magnetic Monopoles in Unified Gauge Theories, Nucl. Phys. B 79 (1974) 276.

[3] A. Polyakov, Particle Specturm in the Quantum Field Theory, JETP Lett 20 (1974) 194.

[4] Y. Cho and D. Maison, Monopole configuration in Weinberg-Salam model, Phys. Lett. B 391 (1997) 360.

[5] T. Kirkman and C. Zachos, Asymptotic Analysis of the Monopole Structure, Phys. Rev. D 24 (1981) 999.

[6] Y. M. Cho, K. Kim and J.H. Yoon, Finite Energy Electroweak Dyon, Eur. Phys. J. C 75, no. 2, 67 (2015) [arXiv:1305.1699 [hep-ph]].

[7] J. Schwinger, Magnetic charge and the charge quantization condition, Phys. Rev. D 12 (1975) 3105 .

[8] S. Ahlen, Stopping-power formula for magnetic monopoles, Phys. Rev. D 17 (1978) 229.

[9] S. Ahlen, Theoretical and experimental aspects of the energy loss of relativistic heavily ionizing particles, Rev. Mod. Phys. 52 (1980) 121.

[10] S. Ahlen and K. Kinoshita, Calculation of the stopping power of very-low-velocity magnetic monopoles, Phys. Rev. D 26 (1982) 2347.

[11] M. Fairbairn, A. Kraan, D. Milstead, T. Sjostrand, P. Skands, and T. Sloan, Stable massive particles at colliders, Phys. Rept. 438 (2007) 1, arXiv:0611040 [hep-ph].

[12] OPAL Collaboration, Search for Dirac magnetic monopoles in $e^{+} e^{-}$collisions with the OPAL detector at LEP2, Phys. Lett. B 663 (2008) 37, arXiv:0707.0404 [hep-ex].

[13] CDF Collaboration, Direct search for Dirac magnetic monopoles in pp collisions at $\sqrt{s}=1.96$ TeV, Phys. Rev. Lett. 96 (2006) 201801, arXiv:0509015 [hep-ex].

[14] J. Pinfold, Searching for the magnetic monopole and other highly ionizing particles at accelerators using nuclear track detectors, Radiat. Meas. 44 (2009) 834.

[15] K. Kinoshita, R. Du, G. Giacomelli, L. Patrizii, F. Predieri, P. Serra, M. Spurio, and J. Pinfold, Search for highly ionizing particles in $e^{+} e^{-}$annihilations at $\operatorname{sqrt}(s)=91.1 \mathrm{GeV}$, Phys. Rev. D 46 (1992) 881.

[16] J. Pinfold, R. Du, K. Kinoshita, B. Lorazo, M. Regimbald, and B. Price, A Search for highly ionizing particles produced at the OPAL intersection point at LEP, Phys. Lett. B 316 (1993) 407. 
[17] M. Bertani, G. Giacomelli, M. Mondardini, B. Pal, L. Patrizii, F. Predieri, P. Serra-Lugaresi, G. Sini, M. Spurio, V. Togo, and S. Zucchelli, Search for Magnetic Monopoles at the Tevatron Collider, Europhys. Lett. 12 (1990) 613.

[18] H1 Collaboration, A direct search for stable magnetic monopoles produced in positron-proton collisions at HERA, Eur. Phys. J. C 41 (2005) 133, arXiv:0501039 [hep-ex].

[19] G. Kalbfleisch, K. Milton, M. Strauss, L. Gamberg, E. Smith, and W. Luo, Improved Experimental Limits on the Production of Magnetic Monopoles, Phys. Rev. Lett. 85 (2000) 5292, arXiv:0005005 [hep-ex].

[20] G. Kalbfleisch, W. Luo, K. Milton, E. Smith, and M. Strauss, Limits on production of magnetic monopoles utilizing samples from the DO and CDF detectors at the Tevatron, Phys. Rev. D 69 (2004) 052002, arXiv:0306045 [hep-ex].

[21] A. De Roeck, A. Katre, P. Mermod, D. Milstead, and T. Sloan, Sensitivity of LHC experiments to exotic highly ionising particles, Eur. Phys. J. C 72 (2012) 1985, arXiv:1112.2999 [hep-ph].

[22] ATLAS Collaboration, Search for magnetic monopoles in sqrt $(s)=7 \mathrm{TeV}$ pp collisions with the ATLAS detector, Phys. Rev. Lett. 109 (2012) 261803, arXiv:1207.6411 [hep-ex].

[23] ATLAS Collaboration, Search for magnetic monopoles and stable particles with high electric charges in $8 \mathrm{TeV}$ pp collisions with the ATLAS detector, CERN-PH-EP-2015-174, arxiv:1509.08059.

[24] MoEDAL Collaboration, Technical Design Report of the Moedal Experiment, CERN-LHCC2009-006 ; MOEDAL-TDR-001 (2009).

[25] B. Acharya et al. [MoEDAL Collaboration], The Physics Programme Of The MoEDAL Experiment At The LHC, Int. J. Mod. Phys. A 29, 1430050 (2014) [arXiv:1405.7662 [hep-ph]].

[26] S. Agostinelli, et al., GEANT4: A Simulation toolkit, Nucl.Instrum.Meth. A506 (2003) 250-303. doi:10.1016/S0168-9002(03)01368-8.

[27] Geant4 Collaboration, http://geant4.cern.ch/support/

[28] A. De Roeck, H.-P. Hächler, A. M. Hirt, M. Dam Joergensen, A. Katre, P. Mermod, D. Milstead, and T. Sloan, Development of a magnetometer-based search strategy for stopped monopoles at the Large Hadron Collider, Eur. Phys. J. C 72 (2012) 2212, arXiv:1206.6793 [physics.ins-det].

[29] B. Acharya et al. [MoEDAL Collaboration], Search for magnetic monopoles with the MoEDAL trapping detector in $8 \mathrm{TeV}$ proton-proton collisions at the LHC, to be submitted (2015).

[30] K. Milton, Theoretical and experimental status of magnetic monopoles, Rep. Prog. Phys. 69 (2006) 1637, arXiv:0602040 [hep-ex]. 